\title{
Characterizing spreading dynamics of subsampled systems with nonstationary external input
}

\author{
Jorge de Heuvel $\odot,{ }^{1}$ Jens Wilting, ${ }^{1}$ Moritz Becker, ${ }^{1,2}$ Viola Priesemann $\odot,{ }^{1, *}$ and Johannes Zierenberg $\odot^{1, *}$ \\ ${ }^{1}$ Max Planck Institute for Dynamics and Self-Organization, 37077 Göttingen, Germany \\ ${ }^{2}$ Department of Computational Neuroscience, Third Institute of Physics-Biophysics, Georg-August-University, 37077 Göttingen, Germany
}

(Received 11 October 2019; revised 24 April 2020; accepted 21 September 2020; published 23 October 2020)

\begin{abstract}
Many systems with propagation dynamics, such as spike propagation in neural networks and spreading of infectious diseases, can be approximated by autoregressive models. The estimation of model parameters can be complicated by the experimental limitation that one observes only a fraction of the system (subsampling) and potentially time-dependent parameters, leading to incorrect estimates. We show analytically how to overcome the subsampling bias when estimating the propagation rate for systems with certain nonstationary external input. This approach is readily applicable to trial-based experimental setups and seasonal fluctuations as demonstrated on spike recordings from monkey prefrontal cortex and spreading of norovirus and measles.
\end{abstract}

DOI: 10.1103/PhysRevE.102.040301

Propagation dynamics in complex networks are often approximated by models with an autoregressive representation. Examples include affinity maturation in immune systems [1], reproductive dynamics of bacteria [2-5], or humans [6], epidemiological disease spreading in a network of humans $[7,8]$, neutron transport theory [9], and collective cortical dynamics [10-15]. The inference of propagation dynamics is often complicated. First, only a fraction of all system components can be observed experimentally (subsampling) [12,16-18]. Second, the model parameters can be time dependent (nonstationary), and specific time-dependent input rates can lead to signatures of criticality even for networks of uncorrelated units [19]. In general, time-dependent input rates are ubiquitous for collective dynamics in neural networks, and are one source for seasonal fluctuations of infectious disease incidence [20].

The subsampling challenge is typically addressed for stationary model parameters. Recent progress has been made for equilibrium and nonequilibrium systems by explicitly modeling the hidden units [21-26]. However, explicit knowledge about the hidden units cannot be guaranteed for real-world applications. A subsampling-invariant approach that does not require knowledge about the underlying model size was recently proposed [12]. The authors showed that established estimators based on linear regression or Kalman filtering underestimate the propagation behavior. They introduced a novel multistep regression (MR) estimator that is subsampling invariant by characterizing propagation dynamics through the systems autocorrelation time $\tau$. However, it does not consider time-dependent model parameters.

*These authors contributed equally to this work.

Published by the American Physical Society under the terms of the Creative Commons Attribution 4.0 International license. Further distribution of this work must maintain attribution to the author(s) and the published article's title, journal citation, and DOI. Open access publication funded by the Max Planck Society.
To tackle nonstationarities, recent approaches considered models with time-dependent parameters. Examples include Bayesian models based on Cox processes [27], weighted least squares [28], or expectation maximization based on Kalman filtering [29,30]. However, none of these methods consider the complication of subsampling, although real spreading processes are usually subsampled [12,31].

In this Rapid Communication, we derive an estimator for a subsampled process subject to a specific type of nonstationary external input, namely, cyclostationary input. We first show that the subsampling-invariant MR estimator [12] can be biased if the external input rate changes over time. We, then, analytically derive a generalization of the MR estimator that can overcome the bias in the case of cyclostationary input. This approach is subsampling invariant and readily applicable to two prevalent situations: First, to trial-based experiments, which are commonly found in neuroscience; second, to periodic input rates, e.g., the seasonal fluctuations of infectious disease incidence [20]. We demonstrate the applicability of our methodology on numerical data (testing robustness to relaxation of our assumptions) and on real-world experimental data.

We consider the class of stochastic processes with an autoregressive representation of first order. This includes widely used processes, such as branching processes and Kesten processes. Time evolves in discrete steps $(\Delta t=1)$. Let $A_{t}^{i}$ denote the activity of a realization $i$ at time $t$, then, the conditional expectation value over the ensemble of independent realizations is defined as

$$
\left\langle A_{t+1}^{i} \mid A_{t}^{i}\right\rangle=m A_{t}^{i}+\left\langle h_{t}\right\rangle,
$$

where $m$ is the time-independent mean offspring parameter and $\left\langle h_{t}\right\rangle$ is the average ensemble rate of a time-dependent input distribution. In the framework of spike propagation in neural networks, $m$ describes the average number of neurons that a single neuron subsequently activates and $\left\langle h_{t}\right\rangle$ describes the expected input rate at time $t$ from sensory modalities or other brain areas. 

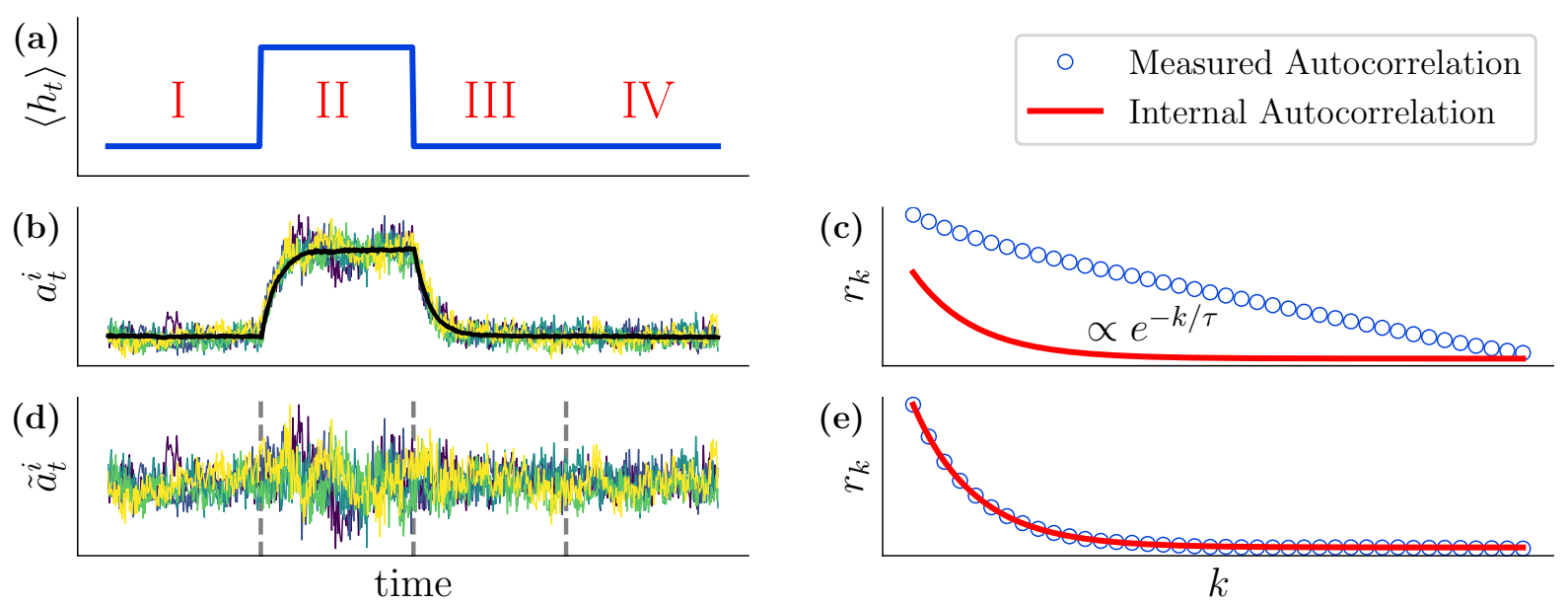

FIG. 1. Unbiased estimation of internal autocorrelation time $\tau$ for a subsampled system with time-dependent input rate can be achieved after subtracting trial-ensemble average from activity. (a) Step function as an example for a time-dependent input rate. (b) Subsampled activity $a_{t}^{i}$ of a branching process with constant internal autocorrelation time $\tau$ and nonstationary input rate shows nonstationary behavior in regimes II and III. The colored lines are individual trials, and the black solid line is the trial-ensemble average $\bar{a}_{t}$ (over 200 trials). (c) Linear regression slope estimates $r_{k}$ (blue dots) of time lag $k$ for the process in (b) do not decay exponentially as expected from the process' internal autocorrelation (red line), which makes an unbiased estimation of $\tau$ impossible. (d) Time series (b) corrected by subtracting trial-ensemble average: $\tilde{a}_{t}^{i}=a_{t}^{i}-\bar{a}_{t}$. (e) For corrected time series (d), the $r_{k}$ decay exponentially, such that $\tau$ can now be inferred without bias. Simulation parameters: Trial length $T=10000$ steps, internal autocorrelation time $\tau=20$ steps, number of trials $N=200$, mean (fully sampled) baseline activity $\left\langle A_{0}\right\rangle=1000$, subsampling fraction $\alpha=0.05$, relative step height $\left\langle h_{\mathrm{up}}\right\rangle /\left\langle h_{\mathrm{down}}\right\rangle=2.6$, and step duration $c=200$ steps.

Note that the expectation values in Eq. (1) are defined over the ensemble of independent realizations (trials) of the stochastic process, e.g., $\left\langle h_{t}\right\rangle=\sum_{i} h_{t}^{i}$ (for the trial average, we drop the index that was summed over). For a general nonstationary external input, $\left\langle h_{t}\right\rangle$ cannot be defined unless one has multiple realizations from the same time-dependent distributions $h_{t}^{i} \sim P\left(h_{t}\right)$. In nature, this is approximately realized by cyclostationarity, e.g., trial-based experiments or seasonal fluctuations. We make use of this to solve the problem even without knowledge of the precise realization of external inputs. In the following, we assume that the generation of offsprings is Poisson distributed with time-independent $m$, whereas the generation of external input is Poisson distributed with time-dependent rate $\left\langle h_{t}\right\rangle$.

Subsampling is incorporated as follows: We only require that the subsampled activity $a_{t}$ is, on average, linear in the full activity $A_{t}^{i}$, i.e., $\left\langle a_{t}^{i} \mid A_{t}^{i}\right\rangle=\alpha A_{t}^{i}$ (for details, see Ref. [12]). For example, every spike or disease incidence is sampled with probability $p=\alpha$.

To estimate the spreading behavior $m$ under subsampling and time-dependent external input rates, we follow the principal idea of the MR estimator [12]. We generalize Eq. (1) by recursive iteration to $k$ time steps,

$$
\left\langle A_{t+k}^{i} \mid A_{t}^{i}\right\rangle=m^{k} A_{t}^{i}+\sum_{l=1}^{k} m^{k-l}\left\langle h_{t+l-1}\right\rangle .
$$

If the rate is time-independent $\left(\left\langle h_{t}\right\rangle=\langle h\rangle\right)$, Eq. (2) implies that the original process $A_{t}^{i}$ has an exponential autocorrelation function,

$$
C(k)=m^{k}=\exp (-k \Delta t / \tau),
$$

with the time lag $k$ in steps of $\Delta t$. The autocorrelation function relates the propagation dynamics $(m)$ to an internal autocorre- lation time $\tau=-\Delta t / \ln (m)$ and represents a measure of how long information persists in the activity [12]. For stationary processes, the variance across trials is equal to the variance within trials $\left[\operatorname{Var}_{i}\left(a_{t}^{i}\right)=\operatorname{Var}_{t}\left(a_{t}^{i}\right)=\operatorname{Var}_{i, t}\left(a_{t}^{i}\right)\right]$ such that the autocorrelation function $C(k)$ of the subsampled activity $a_{t}^{i}$ can be calculated directly via linear regression [12],

$$
C(k)=\hat{r}_{k}=\frac{\operatorname{Cov}_{i, t}\left(a_{t}^{i}, a_{t+k}^{i}\right)}{\operatorname{Var}_{i, t}\left(a_{t}^{i}\right)}=\alpha^{2} \frac{\operatorname{Var}_{i, t}\left(A_{t}^{i}\right)}{\operatorname{Var}_{i, t}\left(a_{t}^{i}\right)} m^{k},
$$

with time-independent autocorrelation strength $b=$ $\alpha^{2} \operatorname{Var}_{i, t}\left(A_{t}^{i}\right) / \operatorname{Var}_{i, t}\left(a_{t}^{i}\right)$ for all $k \neq 0$. Although $b$ is biased under subsampling ( $b<1$ if $\alpha<1$ ), the autocorrelation time $\tau$ is subsampling invariant and can be obtained by fitting Eq. (4) to the data [12].

For a time-dependent external input rate $\left\langle h_{t}\right\rangle$, however, the autocorrelation function is not time invariant, and if calculated does not necessarily decay exponentially [Figs. 1(a)-1(c)]. Consider, for example, a step-function external input rate. Linear regression applied to each regime independently would yield similar slopes (identical slopes for full activity $A_{t}^{i}$ ) but different offsets of linear regression (Supplemental Material [32], Fig. S2). Therefore, the naive application of the MR estimator fails even for full activity. This represents an issue for general time-dependent input.

In the following, we construct a reliable estimate of the internal autocorrelation time $\tau$ in the presence of cyclostationary external input rates. We focus our discussion on subsampled activity $a_{t}$, which includes the fully sampled case $\left(\alpha=1, b_{t}=1\right)$.

To correct the bias from cyclostationary external input $\left(\left\langle h_{t}\right\rangle\right.$ is time dependent but identical for each trial $i$ ), we introduce the following method: Given we have $N$ trials with independent realizations $a^{i}$ of a subsampled linear autoregressive 
process, we calculate the time-dependent trial-ensemble average:

$$
\bar{a}_{t}=\frac{1}{N} \sum_{i=1}^{N} a_{t}^{i},
$$

over all trials (not to be confused with an average calculated over all recorded times). Now, we correct for the nonstationarity of the original process by subtracting the trial-ensemble average [Fig. 1(d)],

$$
\tilde{a}_{t}^{i}=a_{t}^{i}-\bar{a}_{t} .
$$

Its linear regression slopes $r_{k}$ reveal the true internal autocorrelation time in their exponential decay [Fig. 1(e)] for sufficiently large $N$ (see below and Supplemental Material [32] Fig. S4).

From the corrected time series $\tilde{a}_{t}^{i}$, we can, thus, infer the unbiased autocorrelation time by applying the MR estimator [33] (see Supplemental Material [32] Sec. S.6 for the full derivation). To prove this, we reformulate Eq. (4) as simple linear regression at each time across trials, i.e., $\hat{r}_{k, t}=$ $\operatorname{Cov}_{i}\left(\tilde{a}_{t}^{i}, \tilde{a}_{t+k}^{i}\right) / \operatorname{Var}_{i}\left(\tilde{a}_{t}^{i}\right)$. For trial-ensemble corrected $\tilde{a}_{t}^{i}$, we find that the correction compensates the convolution in Eq. (2) such that $\hat{r}_{k, t}=b_{t} m^{k}$ with time-independent decay but with time-dependent autocorrelation strength (Supplemental Material [32] Eq. (S23)),

$$
b_{t}=\alpha^{2} \frac{\operatorname{Var}_{i}\left(A_{t}^{i}\right)}{\operatorname{Var}_{i}\left(a_{t}^{i}\right)} \approx \frac{1}{1-\left(1-\alpha^{-1}\right) F_{t}^{-1}},
$$

where the relation to the (across-trial) Fano factor of the full activity $F_{t}=\operatorname{Var}_{i}\left(A_{t}^{i}\right) /\left\langle A_{t}\right\rangle$ is strictly true only for binomial subsampling. However, we can show (Supplemental Material [32] Eq. (S25)-(S29)) that for the corrected time series, direct application of Eq. (4) with the standard regression approaches yields an unbiased estimate of the internal autocorrelation time $\tau$ despite cyclostationary input and subsampling (for a proof of concept see Fig. S3 of the Supplemental Material [32]).

In addition to the bias from subsampling or nonstationary input, there can be a bias from short trial length $T$ [34] and from small trial number $N$. The short-trial bias can be avoided by estimating both covariance and variance as fluctuations around a global stationary mean (cf., "stationary mean" method in Ref [33] with a detailed discussion). For all our analyses (experimental and numerical), we, thus, use the MR estimator toolbox [33] with the stationary mean method. In principle, this allows for an unbiased estimation down to $N=$ 10 short trials (Fig. S4 of the Supplemental Material [32]), whereas, of course, the variance of the results increases with decreasing $N$ (Supplemental Material [32] Secs. S.4 and S.7).

We tested the applicability of MR estimation for cyclostationary external input by increasing the level of realism for a numerical problem. The test case is a baseline rate $\left\langle h_{0}\right\rangle$ plus step function at onset time $t_{\text {step }}$ with step height $\Delta\langle h\rangle$ and step duration $c$. We consider three cases: (i) perfect cyclostationarity across trials (Fig. 1 and Fig. S5 of the Supplemental Material [32] for an extreme example), (ii) variation of onset time $t_{\text {step }} \sim \mathcal{N}\left(T / 2, \sigma_{t}\right)$ with $\Delta\langle h\rangle=$ $\left\langle h_{0}\right\rangle$ fixed [Fig. 2(a)], and (iii) variation of the step height $\Delta\langle h\rangle \sim \mathcal{N}\left(\left\langle h_{0}\right\rangle, \sigma_{h}\right)$ with $t_{\text {step }}=T / 2$ fixed [Fig. 2(b)]. We (a)

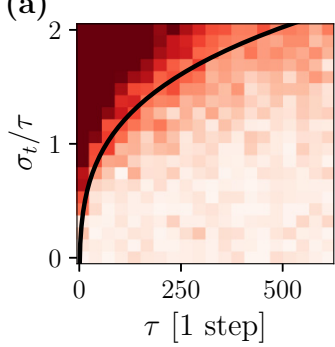

(b)
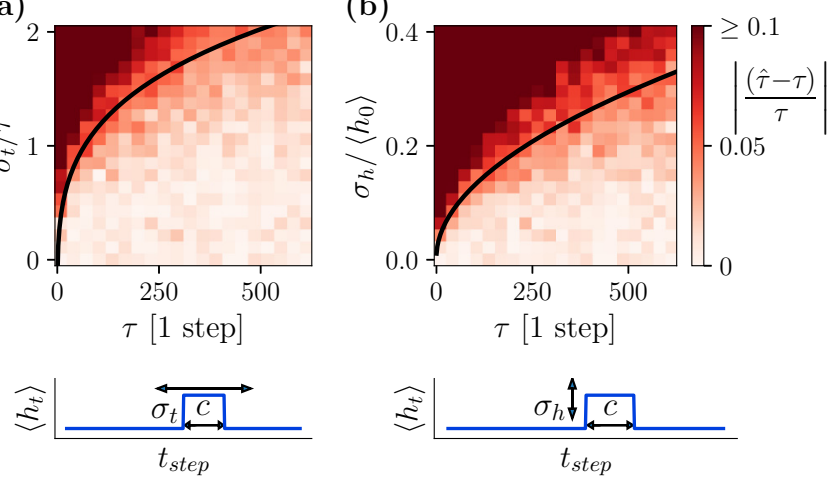

FIG. 2. Robustness of our estimate to variability in a nonstationary input (step function with step-duration $c=10 \tau$ ). (a) Variability in the onset time $t_{\text {step }}$ with standard deviation $\sigma_{t}$. (b) Variability in the step height $\Delta\langle h\rangle$ with standard deviation $\sigma_{h}$. The color in the matrix indicates the relative error between the estimated autocorrelation time $\hat{\tau}$ and the internal autocorrelation time $\tau$ of the branching process. The $5 \%$ error bound was fitted (black lines) and scales as $\sigma \propto \tau^{\gamma}$. Simulation parameters: $T=1000 \tau$ steps, $N=300,\left\langle A_{0}\right\rangle=$ 5000, $\alpha=0.01$.

generated $N=200$ trials of branching processes with internal autocorrelation time $\tau$, trial duration $T=1000 \tau$, and baseline activity $\left\langle A_{0}\right\rangle=5000$ such that $\left\langle h_{0}\right\rangle=(1-m)\left\langle A_{0}\right\rangle(m=$ $\exp (-\Delta t / \tau), \Delta t=1$ step). This setup allows us to independently investigate variability in onset time and height of the input.

Variations in the onset time and step height do not hinder correct inference as long as the standard deviations are sufficiently low (Fig. 2). In our test case, variations in the onset time barely affect the correct inference as long as the standard deviation $\sigma_{t}$ is below the magnitude of the autocorrelation time [Fig. 2(a)]. When $\sigma_{t} \approx O(\tau)$, the method still provides consistent estimates of the processes autocorrelation time. Moreover, the estimates improve for a given $\sigma / \tau$ with increasing autocorrelation time $\tau$. We observe, that the 5\% error bound scales as $\sigma_{t} \propto \tau^{\gamma}$ with $\hat{\gamma} \approx 0.22(3)$. Similarly, variations in the step height barely affect the correct inference as long as the standard deviation $\sigma_{h}$ is below $\left\langle h_{0}\right\rangle / 5$ [Fig. 2(b)]. Again, the estimates improve with increasing autocorrelation time and the $5 \%$ error bound scales as $\sigma_{h} / \Delta\langle h\rangle \propto \tau^{\gamma}$ with $\hat{\gamma} \approx 0.4(1)$. We conclude that our method provides consistent results even after relaxation of perfect cyclostationarity.

We applied our method to two sets of experimental data. The first dataset consists of spiking activity in prefrontal cortex from a trial based short-term visual memory task on macaque mulatta [35] (about $N=300$ trials each, see Supplemental Material [32] Sec. S.11). In this dataset, the external input can be interpreted as sensory input from other brain areas to the investigated area. The second dataset are epidemiological case reports from the Robert Koch Institute [36] ( $N=18$ trials each, see Supplemental Material [32] Sec. S.10). In the epidemiological dataset, the infections carried into the country via travel can be interpreted as nonstationary external input.

For the monkey data, we want to emphasize three findings: First, although the trial-ensemble average $\bar{a}_{t}$ increases by a 

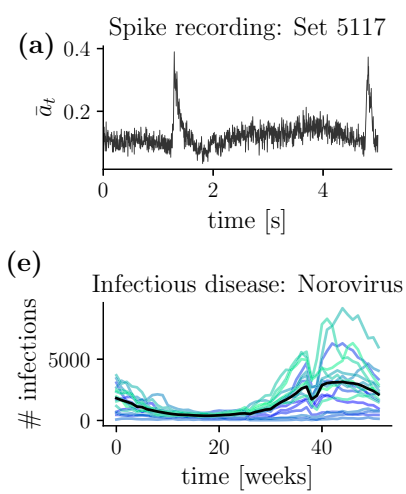

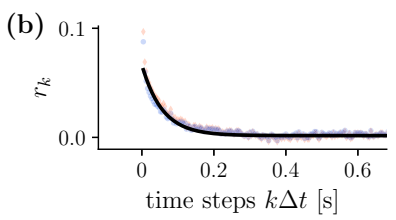

$$
\text { (c) } 400-
$$

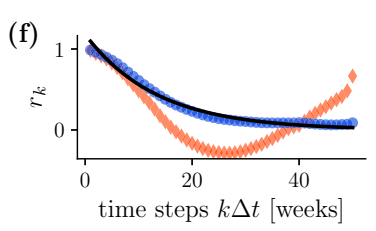

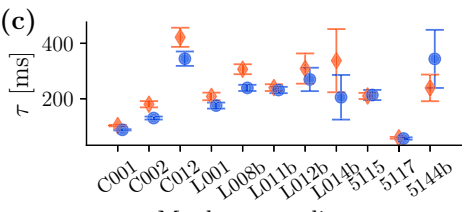

Monkey recording set

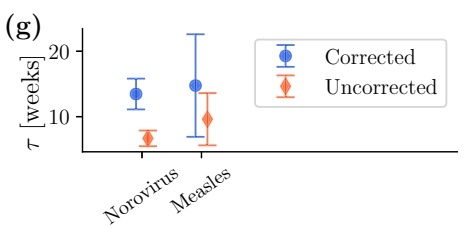

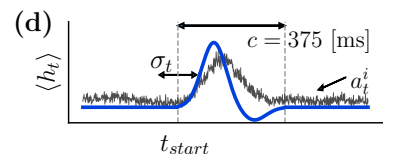

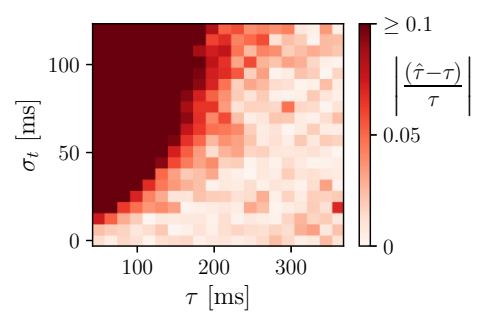

FIG. 3. Application of our new approach to experimental data. Top (a)-(c) The intrinsic timescales $\tau$ in the macaque prefrontal cortex have been inferred with our new approach from spike recordings during a trial-based visual short-term memory task [35]. (a) Example trial-ensemble average of stimulus-evoked nonstationary neural responses. (b) Autocorrelation functions $r_{k}$ of (a) before (orange) and after the correction (blue) hardly differ. (c) Intrinsic timescales inferred from uncorrected data are systematically but not very strongly overestimated (less than $10 \%$ ). (d) Numerical robustness validation for typical experimental recordings, resembling a typical evoked potential: Nonstationary external input (blue) with twofold increase during stimulus presentation (total duration of $c=375 \mathrm{~ms}$ ) for $N=300$ trials of length $T=5 \mathrm{~s}$ with sampling frequency $f=1 \mathrm{kHz}$ and subsampling fraction $\alpha=0.01$. An example trial realization $a_{t}^{i}$ is shown as a black line. The impact on estimating $\tau$ under variance of the stimulus onset $\left(\sigma_{t}\right)$ is evaluated for various intrinsic autocorrelation times $\tau$ as in Fig. 2. Bottom (e)-(g) The infectious spreading dynamics of norovirus and measles have been inferred with case report data from the Robert Koch Institute [36]. (e) Reported infection numbers (blue lines) and the time-dependent trial-ensemble average (black line) for norovirus reveal seasonal nonstationarities. (f) With our method, the seasonality was mostly removed from the autocorrelation function $r_{k}$ of (d). (g) In contrast to the neural recordings, the infectious spreading dynamics inferred from the uncorrected disease data are systematically underestimated.

factor of 3 [Fig. 3(a)], the autocorrelation function hardly differs in most cases [Fig. 3(b)]. Second, we find a systematic decrease in intrinsic timescales after the correction, whereas, for the majority of the recording sets, the decrease was less than $10 \%$ [Fig. 3(c)]. Third, a robustness test of our method with parameters adjusted to experimental scale [Fig. 3(d) with experimentally realistic stimulus shape] indicates that our method yields less than 5\% deviation from $\tau \geqslant 200 \mathrm{~ms}$ despite stimulus onset variability with $\sigma_{t}<50 \mathrm{~ms}$, which is a realistic constraint given the steep rise of typical ensemble responses within 30-50 ms [Fig. 3(a)]. To conclude, our method reveals intrinsic timescales in the prefrontal cortex between 57(4) and 345(26) ms with median $214 \mathrm{~ms}$ (compared to $239 \mathrm{~ms}$ if not corrected) from recordings covering the full task. Our results are consistent with previous results in the prefrontal areas of the macaque (about $200 \mathrm{~ms}$ ) confined to the stimulus foreperiod to approximate the resting state $[37,38]$.

In the example of disease spreading, our method accounts well for seasonal fluctuations [Figs. 3(e)-3(g)]. The weekly case number reports reveal a strong yearly periodicity, suggesting a yearwise separation into trials. The improvement due to the trial-ensemble average correction is readily visible in the regression function $r_{k}$ [Fig. 3(f)]. With the correction, the infectiousness estimate is higher than without [Fig. $3(\mathrm{~g})$, norovirus: $\tau=14(3)$ weeks, measles: $\tau=15(8)$ weeks]. The disease results are, in principle, subject to additional uncertainty from the small number of trials (cf., Fig. S4 of the Supplemental Material [32]), which are probably on the order of $10 \%$ and, thus, smaller than the error bars from the fits. Our results highlight that the correction by trial-ensemble average can reveal higher infectiousness of diseases, which might otherwise be underestimated due to seasonal fluctuations and other nonstationary effects, and that long-term recordings are necessary to reveal the intrinsic infectiousness of a disease.

To summarize, we have presented a simple subsamplinginvariant estimate of the internal autocorrelation time for stochastic processes with an autoregressive representation subject to (approximate) cyclostationary external input. The key success of the presented approach (MR estimation with trial-ensemble average corrected time series) is the potential to disentangle the internal spreading from any hidden but repetitive external input rate. Thereby, our approach solves the problem of apparent criticality due to nonstationary input rates [19] for repetitive stimulation protocols. We demonstrated the robustness of our approach to violations of perfect cyclostationarity for the external input rate; and we showed its applicability to real-world problems from neuroscience and epidemiology. In conclusion, we recommend the trial-ensemble average correction as the best practice when approximating trial-based experiments with autoregressive models. A toolbox for the multistep-regression analysis is readily available [33].

We thank M. Munk for sharing his data. All authors acknowledge support by the Max Planck Society. Financial support was received from the Gertrud-Reemtsma-Stiftung (J.W.), the Joachim Herz Stiftung (J.Z.), and the German Ministry of Education and Research $(\mathrm{BMBF})$ via the Bernstein Center for Computational Neuroscience (BCCN) Göttingen under Grant No. 01GQ1005B (M.B., V.P., and J.Z.).
[1] A. Nourmohammad, J. Otwinowski, M. Łuksza, T. Mora, and A. M. Walczak, Mol. Biol. Evol. 36, 2184 (2019).
[2] B. H. Good and O. Hallatschek, Curr. Opin. Microbiol. 45, 203 (2018). 
[3] D. G. Kendall, J. R. Stat. Soc., Ser, B (Methodological) 11, 230 (1949).

[4] M. A. Muñoz, Rev. Mod. Phys. 90, 031001 (2018).

[5] Y. Kozlovsky, I. Cohen, I. Golding, and E. Ben-Jacob, Phys. Rev. E 59, 7025 (1999).

[6] I. M. del Puerto, M. González, C. Gutiérrez, R. Martínez, C. Minuesa, M. Molina, M. Mota, and A. Ramos (eds.), Branching Processes and Their Applications, Lecture Notes in StatisticsProceedings (Springer, Berlin, Heidelberg, 2016).

[7] C. P. Farrington, M. N. Kanaan, and N. J. Gay, Biostatistics 4, 279 (2003).

[8] O. Diekmann, J. A. P. Heesterbeek, and J. A. J. Metz, J. Math. Biol. 28, 365 (1990).

[9] A. Pazy and P. H. Rabinowitz, Arch. Ration. Mech. Anal. 51, 153 (1973).

[10] J. M. Beggs and D. Plenz, J. Neurosci. 23, 11167 (2003).

[11] C. Haldeman and J. M. Beggs, Phys. Rev. Lett. 94, 058101 (2005).

[12] J. Wilting and V. Priesemann, Nat. Commun. 9, 2325 (2018).

[13] J. Zierenberg, J. Wilting, V. Priesemann, and A. Levina, Phys. Rev. E 101, 022301 (2020).

[14] J. P. Neto, F. P. Spitzner, and V. Priesemann, biorXiv:759613v2 (2019).

[15] A. Hagemann, J. Wilting, B. Samimizad, F. Mormann, and V. Priesemann, arXiv:2004.10642.

[16] V. Priesemann, M. H. Munk, and M. Wibral, BMC Neurosci. 10, 40 (2009).

[17] T. L. Ribeiro, M. Copelli, F. Caixeta, H. Belchior, D. R. Chialvo, M. A. L. Nicolelis, and S. Ribeiro, PLoS One 5, e14129 (2010).

[18] A. Levina and V. Priesemann, Nat. Commun. 8, 15140 (2017).

[19] V. Priesemann and O. Shriki, PLoS Comput. Biol. 14, e1006081 (2018).

[20] J. E. Franke and A.-A. Yakubu, SIAM J. Appl. Math. 66, 1563 (2006).

[21] B. Bravi, M. Opper, and P. Sollich, Phys. Rev. E 95, 012122 (2017).
[22] B. Dunn and Y. Roudi, Phys. Rev. E 87, 022127 (2013).

[23] B. Bravi and P. Sollich, J. Stat. Mech.: Theory Exp. (2017) 063404.

[24] L. Bachschmid-Romano and M. Opper, J. Stat. Mech.: Theory Exp. (2014) P06013.

[25] B. Dunn and C. Battistin, J. Phys. A: Math. Theor. 50, 124002 (2017).

[26] A. Das and I. R. Fiete, biorXiv:512053v1 (2019).

[27] C. Donner and M. Opper, J. Mach. Learn. Res. 19, 1 (2018).

[28] T. S. Rao, J. R. Stat. Soc.: Series B (Methodological) 32, 312 (1970).

[29] Z. Ghahramani and G. E. Hinton, Parameter Estimation for Linear Dynamical Systems, Technical Report No. CRG-TR-96-2, University of Toronto, Dept. of Computer Science, 1996.

[30] R. H. Shumway and D. S. Stoffer, J. Time Ser. Anal. 3, 253 (1982).

[31] L. Papoz, B. Balkau, and J. Lellouch, Int. J. Epidemiol. 25, 474 (1996).

[32] See Supplemental Material at http://link.aps.org/supplemental/ 10.1103/PhysRevE.102.040301 for detailed analytic derivations, additional tests for extreme cases of time-dependent input rates that complement examples in the main paper, additional proof of principles for subsampling and finite trial numbers, as well as additional information regarding the experimental data.

[33] F. P. Spitzner et al., arXiv:2007.03367 (2020).

[34] F. H. C. Marriott and J. A. Pope, Biometrika 41, 390 (1954).

[35] G. Pipa, E. S. Städtler, E. F. Rodriguez, J. A. Waltz, L. Muckli, W. Singer, R. Goebel, and M. H. J. Munk, Front. Integr. Neurosci. 3, 25 (2009).

[36] SurvStat@RKI 2.0, https://survstat.rki.de/, accessed: 2019-0107.

[37] J. D. Murray, A. Bernacchia, D. J. Freedman, R. Romo, J. D. Wallis, X. Cai, C. Padoa-Schioppa, T. Pasternak, H. Seo, D. Lee, and X.-J. Wang, Nat. Neurosci. 17, 1661 (2014).

[38] R. Chaudhuri, K. Knoblauch, M.-A. Gariel, H. Kennedy, and X.-J. Wang, Neuron 88, 419 (2015). 